\title{
Effect of Crystal Boundaries on the Initiation of Fatigue Cracks in Aluminum Bicrystals*
}

\author{
By Genjiro Mima**, Fukuji Inoko*** \\ and Ken Atagi**
}

\begin{abstract}
Four types of aluminum bicrystals having a longitudinal twist boundary parallel to the $\{111\}$ plane and with a misfit angle of about 0.38 rad $\left(22^{\circ}\right)$ were grown by a modified Bridgman method. The fatigue tests were done on a bending fatigue testing machine and carried out at a constant strain amplitude of about $0.2 \%$ and cyclic frequencies of $30.4 \mathrm{~Hz}$.

It has been found that fatigue cracks are initiated along the crystal boundaries. The initiation of the fatigue cracks along the crystal boundaries is due to the operation of the secondary slip systems whose slip planes are parallel to the crystal boundary one.
\end{abstract}

(Received September 3, 1979)

\section{Introduction}

It has been reported that fatigue cracks are initiated in persistent slip bands within grains in aluminum and other alloys ${ }^{(1)}$. Fatigue cracks have also been observed to initiate along grain boundaries in polycrystals, for example, in aluminum by $\mathrm{Smith}^{(2)}$, in copper by Kemsley ${ }^{(3)}$, in nickel by Laird et al. ${ }^{(4)}$, and in steel by Wood et al. ${ }^{(5)}$ In the latter case, however, the initiation mechanism of the fatigue cracks has not yet been examined systematically. The authors have examined systematically the effect of the crystal boundaries on the tensile deformation in many types of aluminum bicrystals ${ }^{(6)}$ (9). They also have reported that fatigue cracks are initiated in the crystal boundary affectedregions in aluminum bicrystals under the following conditions: (1) $N_{i j}$ values $^{(10)}$ between the primary slip systems in both component crystals are small, and (2) dislocations of either of the primary slip systems or both of those in two component crystals have a large screw component compared with the edge one ${ }^{(11)}$.

In the present work, the effect of the crystal

* This paper was published originally in Japanese in Journal of the Japan Inst. of Metals, 42 (1978), 887.

** Anan Technical College, Anan 774, Japan.

*** Department of Production Mechanics, Technical College, The University of Tokushima, Tokushima 770, Japan. boundaries on the initiation of fatigue cracks along the crystal boundaries have been studied with use of aluminum bicrystals (Fig. 1) which satisfy the condition (2). In these bicrystals, dislocations of both of the primary slip systems in two component crystals possess pure screw

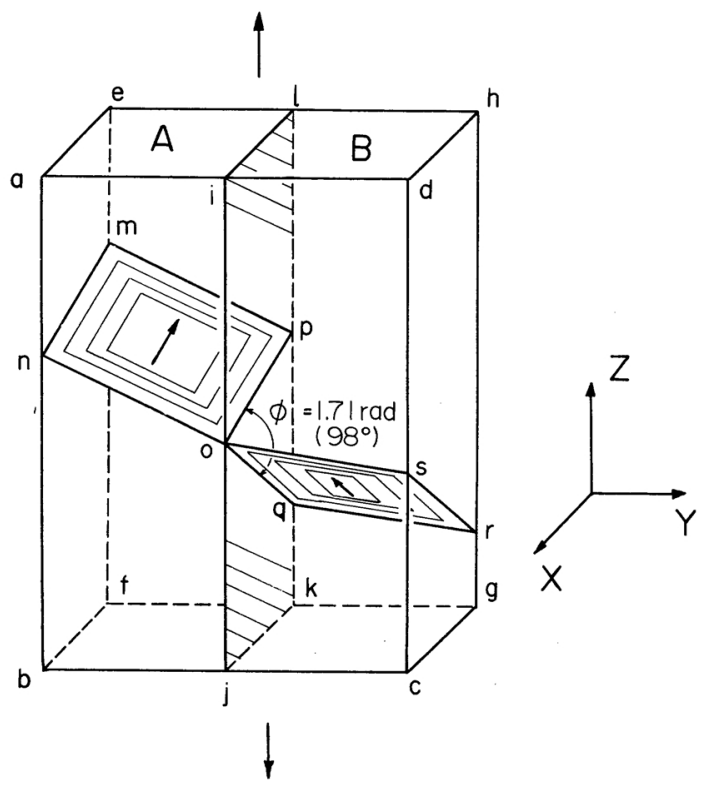

Fig. 1 Schematic drawing of a bicrystal (type 3) with a $0.38 \mathrm{rad}\left(22^{\circ}\right)$ twist crystal boundary i-j-k-l whose plane is $\{111\}$. The surface $a-b-c-d$ is called the front surface and the e-f-g-h the back one. The planes m-n-o-p and o-q-r-s are primary slip planes in two component crystals $\mathrm{A}$ and $\mathrm{B}$, respectively. Both slip vectors intersect mutually at $1.71 \mathrm{rad}$ $\left(98^{\circ}\right)$ on the crystal boundary plane. 
components along a certain direction on each crystal boundary plane.

\section{Experimental Procedures}

Four types of $99.99 \mathrm{wt} \%$ aluminum bicrystals with a longitudinal twist boundary, whose misfit angle was about $0.38 \mathrm{rad}\left(22^{\circ}\right)$, parallel to the $\{111\}$ plane were grown by a modified Bridgman method. Orientations of their tensile axes in component crystals $\mathrm{A}$ and $\mathrm{B}$ are shown in Fig. 2. The mark 1A signifies the orientation for the tensile axis of component crystal $A$ in type 1 bicrystal. $P$ and $D$ signify $\langle 111\rangle$ and $\langle 110\rangle$, respectively. The errors between each orientation expected and the one obtained were less than $\pm 0.05 \mathrm{rad}\left( \pm 3^{\circ}\right)$. Eight fatigue specimens (two for four types of bicrystals, respectively) were prepared. Their dimensions are shown in Fig. 3. The stress concentration factor $\alpha$ is $1.09^{(12)}$. They were annealed at $573 \mathrm{~K}$ for $10.3 \mathrm{ks}$, electropolished in a solution of 1 part perchloric acid and 4 parts acetric acid, and then tested under the conditions of a cyclic frequency of $30.4 \mathrm{~Hz}$ and a constant strain of about $0.2 \%(\mathrm{R}=-1)$ by a Shenk type bending fatigue testing machine newly devised $^{(11)}$. The development of slip bands and fatigue cracks were observed by an optical microscope at the interruptions of the test. In the present work, the stress condition on the gage length of the specimen tested by the Schenk type bending is regarded as that by a four-point bending.

\section{Results}

It has been confirmed as the reproductible facts that fatigue cracks are initiated along each crystal boundary on the front surfaces (Fig. 1) in type 3 and 4 bicrystals. Stereographic projections of component crystals $A$ and $B$ in type 3 are shown in Fig. 4. For example, the mark P3D $\overline{3} A$ shows the slip system in which the slip plane is $P 3$ and the direction is $D \overline{3}$ in component crystal $\mathrm{A} . \mathrm{D} 3$ and $\mathrm{D} \overline{3}$ give two mutually reverse slip directions, respectively. The solid lines represent the traces of the primary slip systems P3D3-D $\overline{3} A$ and P1D4$\mathrm{D} \overline{4} \mathrm{~B}$, and the dotted lines, those of the secon-

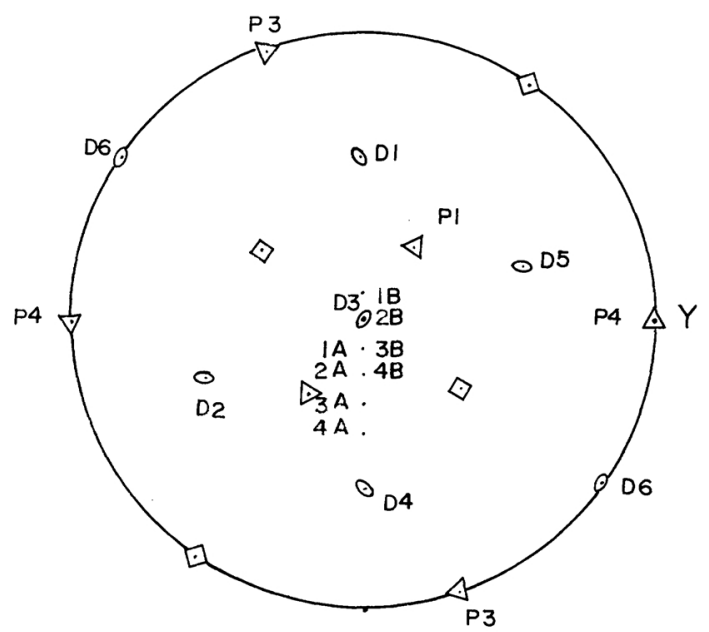

Fig. 2 Tensile axes of two component crystals $A$ and $B$ in four types of bicrystals.

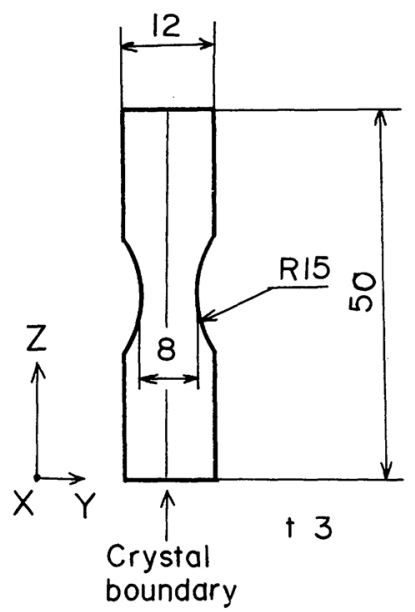

Fig. 3 Dimensions of fatigue specimens.

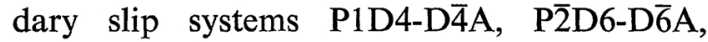

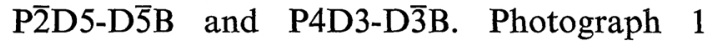
shows the development of the slip bands near the crystal boundary in type 3 with an increase of the number of cycles. At the first cycle and $10^{2}$ cycles, the slip bands of P3D3-D $\overline{3} A$ diminish with the approach toward the crystal boundary. This is probably due to the repulsive force between the screw dislocations of the slip system P3D3-D $\overline{3} A$ and the dislocation walls formed by the screw dislocations of the slip

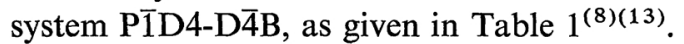

On the back surface of the same bicrystal, the slip bands of the primary slip systems P3D3- 


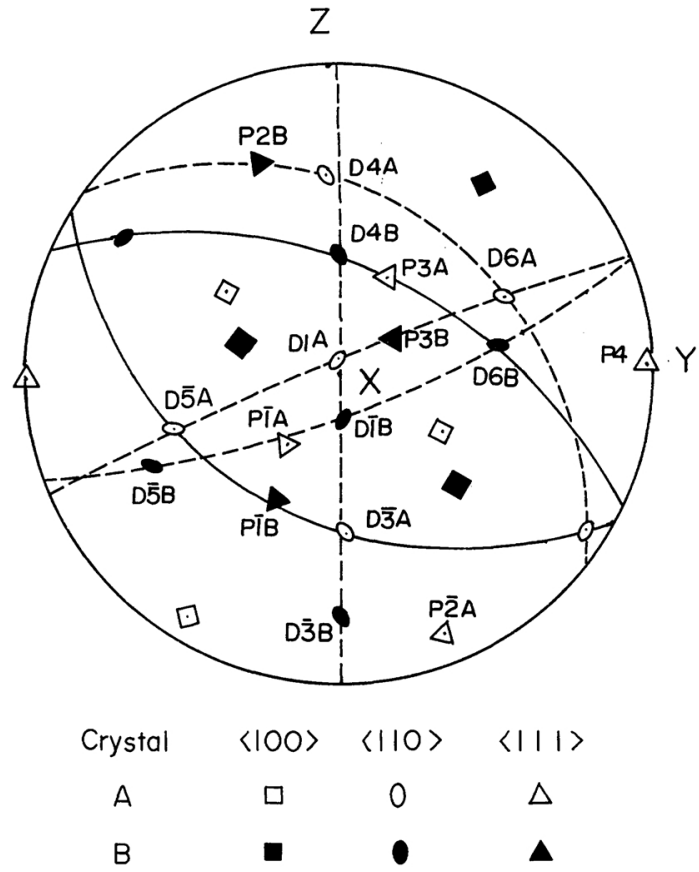

Fig. 4 Stereographic projections of two component crystals A and B in type 3 bicrystal. Solid and dotted lines show traces of the primary and secondary slip systems, respectively.

$\mathrm{D} \overline{3} \mathrm{~A}$ and P$\overline{1} \mathrm{D} 4-\mathrm{D} \overline{4} \mathrm{~B}$, and secondary ones $\mathrm{P} \overline{2} \mathrm{D} 6-\mathrm{D} \overline{6} \mathrm{~A}$ and P2D5-D $\bar{B} \mathrm{~B}$ are observed near the crystal boundary, respectively. These secondary ones cannot be observed on the front surface. The slip bands of P4D3-D鸟, whose slip planes parallel to the crystal boundary plane and Schmid factor is about zero, are activated over about $40 \mu \mathrm{m}$ from the crystal boundary. The slip bands of the primary and secondary slip systems develop with increasing number of cycles. A micro fatigue crack is observed on the front surface at $1.2 \times 10^{4}$ cycles in type 3, as shown in Photo. 2. A long fatigue crack along the crystal boundary at $1.5 \times$ $10^{4}$ cycles is shown in Photo. 3. This long fatigue crack is formed by connecting the short fatigue cracks which are separately initiated and propagated to a length of about $1 \mathrm{~mm}$ along the crystal boundary. After the long fatigue crack has grown to about $5 \mathrm{~mm}$ along the crystal boundary, two fatigue cracks branch off from it and propagate into component crystals A and B (Photo. 4). Finally, another crack initiates in a primary slip band on an edge of component crystal A. Then, it propagates by taking a zigzag course along the same sort of the slip bands, and leads to fracture at $1.8 \times 10^{5}$ cycles as shown in Photo. 5. It can be recognized that in Photo. 6 the shapes of etch-pits formed on a cross section of the specimen are different on both sides of the fatigue crack, which propagates about $1 \mathrm{~mm}$ into the interior of the specimen. This means that fatigue crack propagates not only toward the longitudinal direction, but also into the interior of the specimen along the crystal boundary.

The behavior of slip bands and the initiation and propagation of fatigue cracks during the fatigue test for type 4 are similiar to that for type 3. Namely, on the front surface of type 4 the slip bands of P4D3-D $\overline{3} B$ with slip planes parallel to the crystal boundary plane are activated remarkably near the crystal boundary in component crystal $\mathrm{B}$, and micro fatigue

Table 1 The interaction force between the screw dislocation wall and approaching screw dislocations in four types of bicrystals (under tensile stress).

\begin{tabular}{|c|c|c|c|c|}
\hline Type & Screw dislocation wall & Approaching screw dislocation & $\phi \operatorname{rad}\left(^{\circ}\right)$ & Direction of force \\
\hline \multirow{4}{*}{$\begin{array}{c}1 \\
{[2]}\end{array}$} & P̄̄D4A & P2D1̄B & $1.66 \quad(95)$ & \multirow[t]{2}{*}{ Attractive } \\
\hline & Left-handed & Left-handed & [1.72] (99) & \\
\hline & $\overline{\mathrm{P}} \overline{1} \mathrm{D} \overline{4 \mathrm{~A}}$ & P1D4B & $2.70(155)$ & Attractive \\
\hline & Left-handed & Right-handed & [2.77] (159) & \\
\hline \multirow{6}{*}{$\begin{array}{c}3 \\
{[4]}\end{array}$} & P1̄D4B & $\mathrm{P} 3 \mathrm{D} \overline{3} \mathrm{~A}$ & 1.71 & \multirow[t]{2}{*}{ Repulsive } \\
\hline & Right-handed & -(Right-handed) & {$[1.71]$} & \\
\hline & PIDD4B & $\mathrm{P} \overline{2} \mathrm{D} 6 \mathrm{~A}$ & 0.94 & \multirow[t]{2}{*}{ Attractive } \\
\hline & Right-handed & Right-handed & {$[0.92]$} & \\
\hline & $\mathrm{P} 3 \mathrm{D} \overline{3} \mathrm{~A}$ & $\mathrm{P} 2 \mathrm{D} \overline{\mathrm{B}}$ & 0.94 & \multirow[t]{2}{*}{ Attractive } \\
\hline & -(Right-handed) & -(Right-handed) & [0.92] & \\
\hline
\end{tabular}




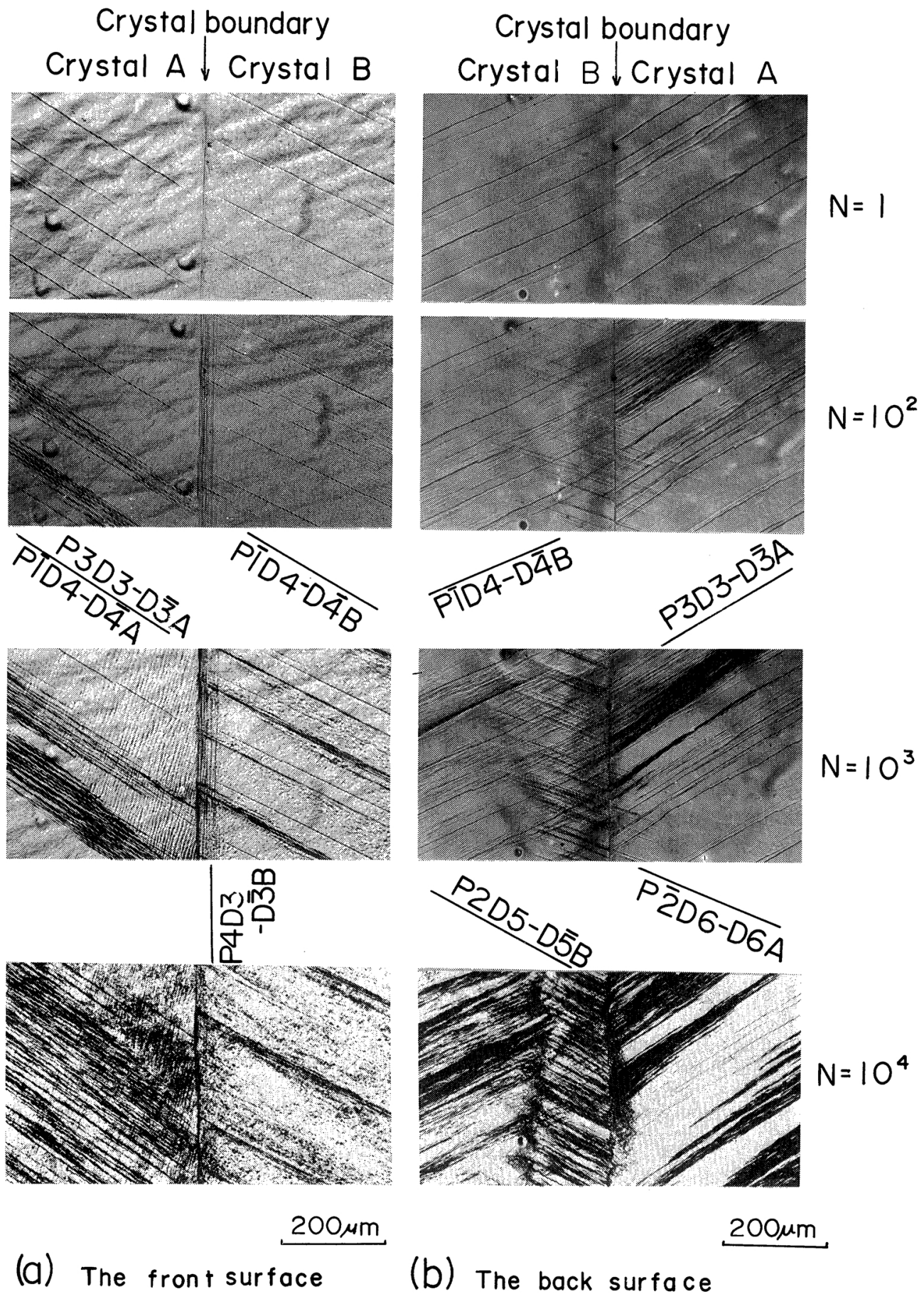

Photo. 1 Development of slip bands near the crystal boundary in type 3 bicrystal with an increase of the number of cycles. 


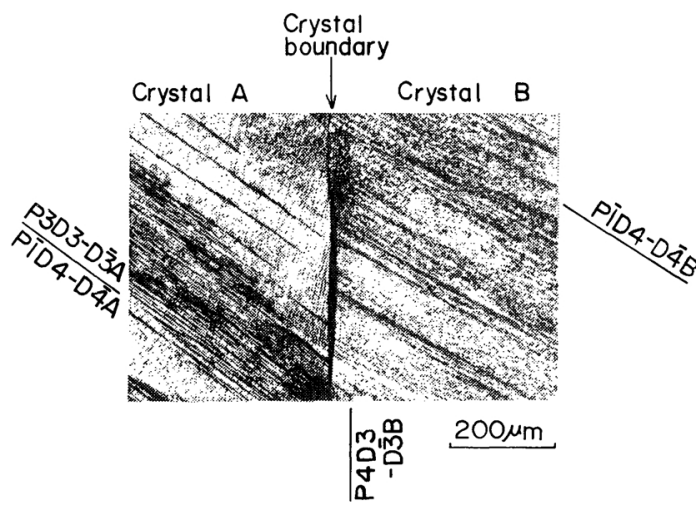

Photo. 2 A fatigue microcrack initiated along the crystal boundary at $N=1.2 \times 10^{4}$ in type 3 bicrystal.

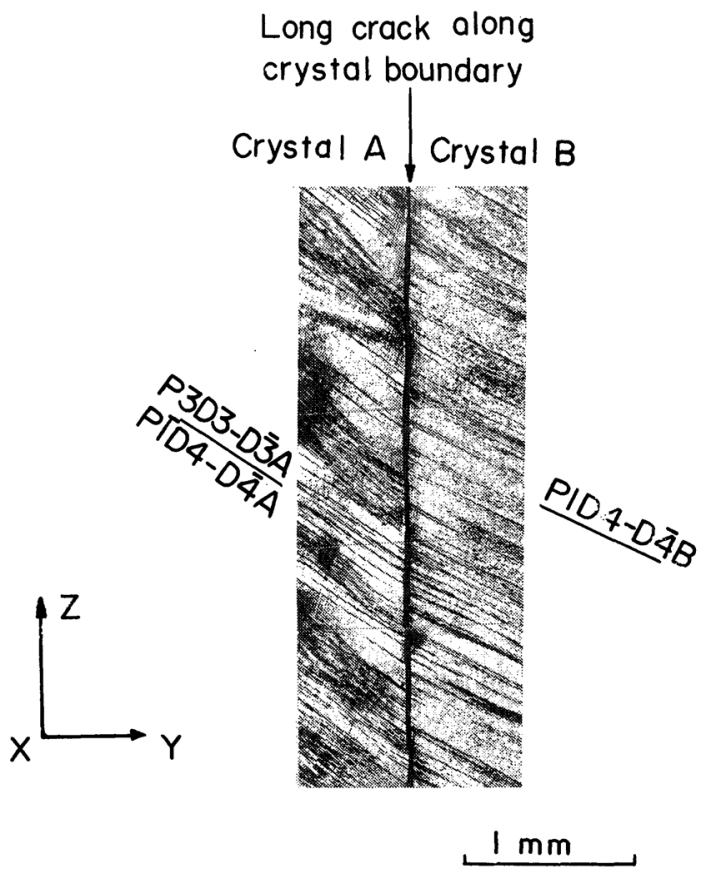

Photo. 3 A long fatigue crack grown by connecting short cracks along the crystal boundary at $N=1.5 \times$ $10^{4}$ in type 3 bicrystal.

cracks along the crystal boundary are initiated at $10^{5}$ cycles. On the back surface any fatigue crack along the crystal boundary is not initiated.

On the other hand, in type 1 and 2 no fatigue cracks along the crystal boundary are initiated on either surface. Stereographic projections of component crystals A and B in type 1 are shown in Fig. 5. The solid lines represent the traces of the primary slip systems $\bar{P} \overline{1} \mathrm{D} 4-\mathrm{D} \overline{4} \mathrm{~A}$ and

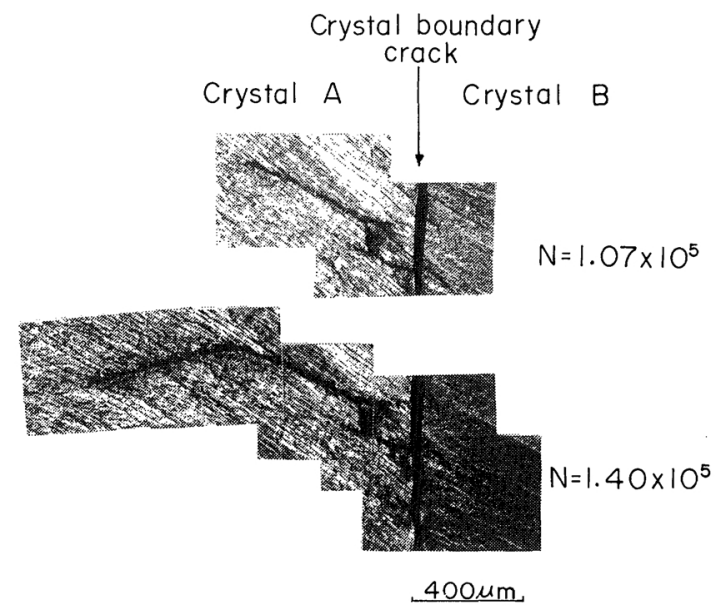

Photo. 4 A fatigue crack propagated into two component crystals from the crystal boundary crack in type 3 bicrystal.

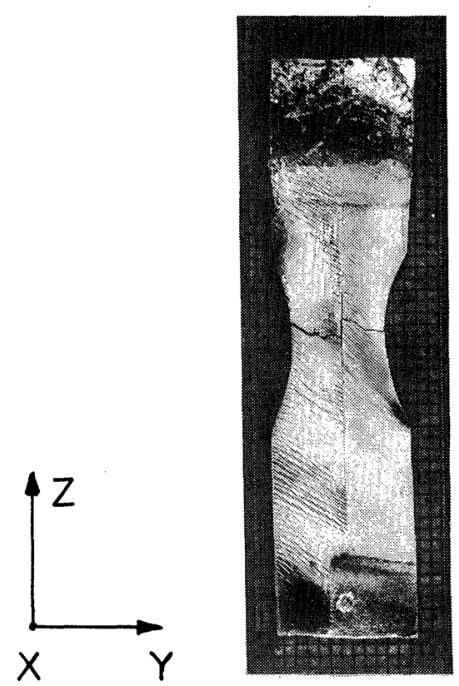

Photo. 5 Microphotograph of the specimen fractured at $N=1.8 \times 10^{5}$ in type 3 bicrystal. The main crack is nucleated at the left corner and propagates along the primary slip bands. Then, it is connected with the crystal boundary crack which have already initiated, and then propagates into the right crystal.

P2D1-D1B. The appearance of the slip bands near the crystal boundary on the front surface in type 1 at 500 cycles is shown in Photo. 7. The primary slip systems P $\overline{1} D 4-D \overline{4} A$ and P2D1-DīB are activated, but the slip system P4D3-D鸟 with slip planes parallel to the crystal boundary plane is not. The slip band tips of P2D1-DīB reach the crystal boundary. 


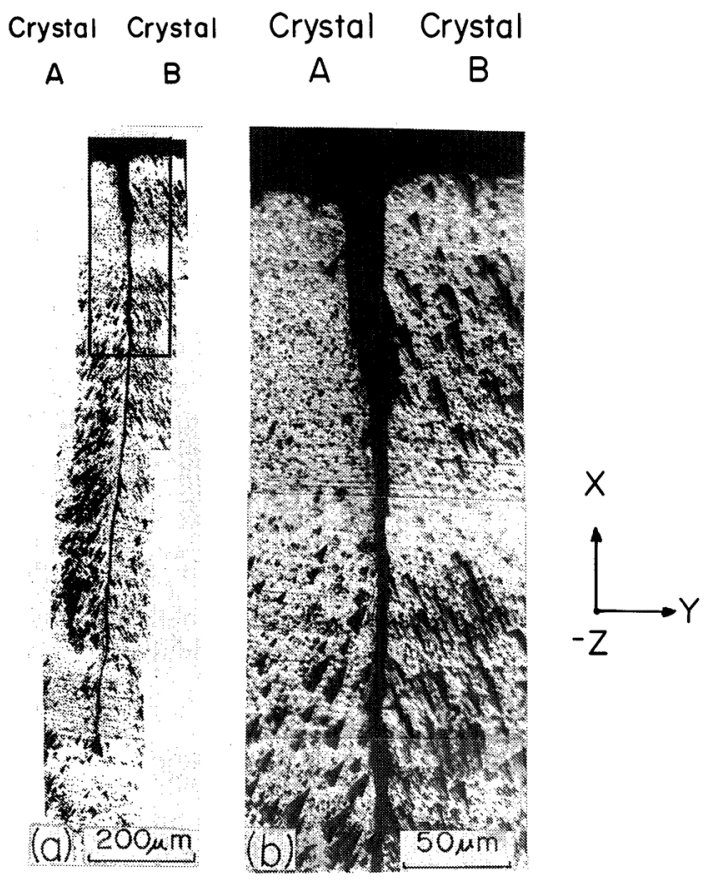

Photo. 6 Shape of crack on a cross section of the type 3 bicrystal. It is recognized that the fatigue crack propagates along the crystal boundary not only on the specimen surface but also into the interior of the specimen.

This can be expected from Table 1 in which the screw dislocation walls formed on the crystal boundary by the primary slip system P1D4-D $\overline{4}$ A may exert the attractive force over the long range upon the screw dislocations of the primary slip system P2D1-D1B. The development of the primary slip bands remarkably increases with an increase of the number of cycles. But no fatigue cracks along the crystal boundary are observed. The main fatigue crack which leads the specimen to final fracture is initiated in the primary slip bands on an edge of the specimen and propagates along the same sort of slip bands.

The development of slip bands during the fatigue test in type 2 is similar to that in type 1 . In type 2 no fatigue cracks along the crystal boundary are observed on either surface.

\section{Discussion}

It has been confirmed that the fatigue cracks

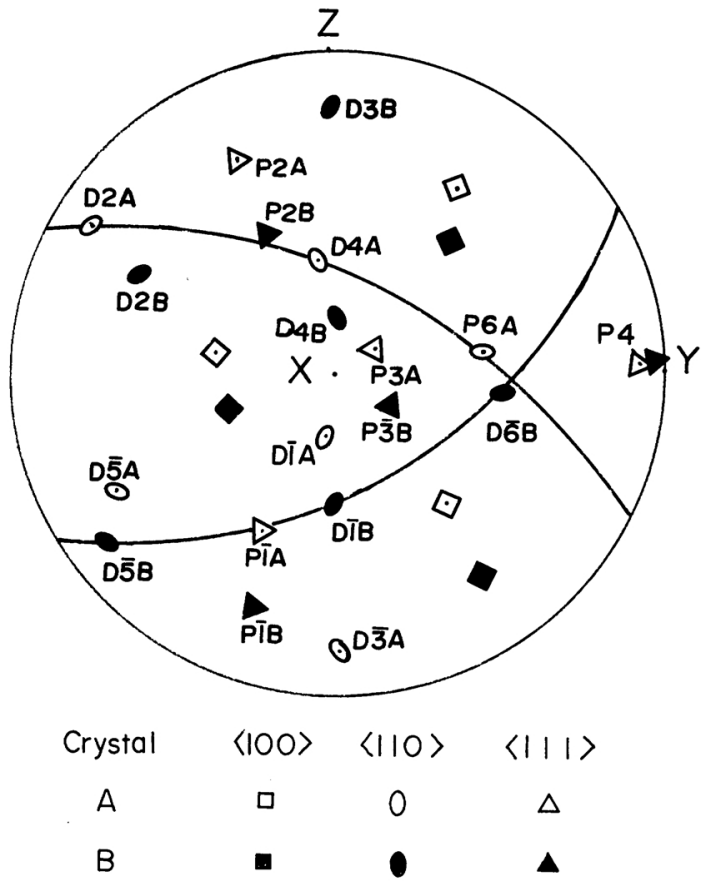

Fig. 5 Stereographic projections of two component crystals A and B in type 1 bicrystal. Solid lines show traces of the primary slip system in both crystals.

along the crystal boundaries are initiated on the front surfaces in type 3 and 4 bicrystals, but not on the back surfaces of the same bicrystals and on neither surface in type 1 and 2 . Near the crystal boundaries on the front surfaces in type 3 and 4 , the slip bands of P4D3-D 3 B, whose slip planes are parallel to the crystal boundary plane and whose Schmid factor is nearly zero are remarkably activated. Then the fatigue cracks are initiated along the crystal boundaries at which the slips of P4D3-D $\overline{3} B$ are most remarkable. Here, the activation of P4D3-D $\overline{3} B$ slips suggests that the local shear stresses $\pm \tau_{y z}$ act near the crystal boundaries.

Generally, if component crystals A and B maintain the compatibility requirements of elastic strains at the crystal boundary, the elastic strain $e_{e x x}, e_{z z}$ and $e_{e} \gamma_{x z}$ must satisfy the following equations:

$$
e^{\varepsilon_{x x}^{A}}=\stackrel{B}{B}, e_{e x z}^{A}=e_{e}^{\varepsilon_{z z}^{B}},{ }_{e} \gamma_{x z}^{A}={ }_{e} \gamma_{x z}^{B} .
$$

If a bicrystal with the incompatibility of the elastic strains is deformed under the condition maintaining the compatibility requirements of 


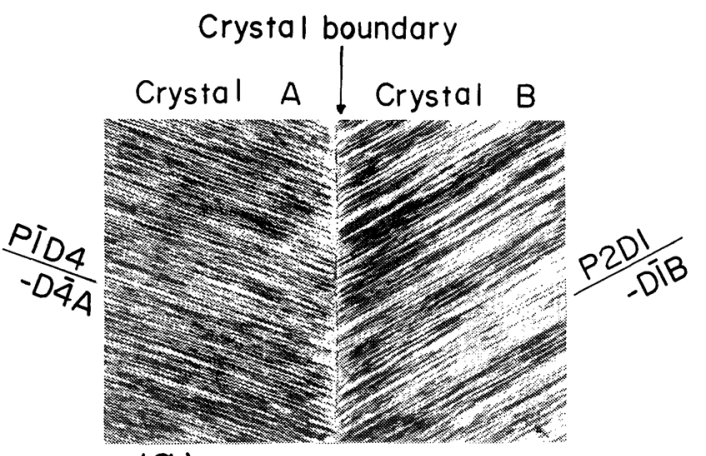

(a)

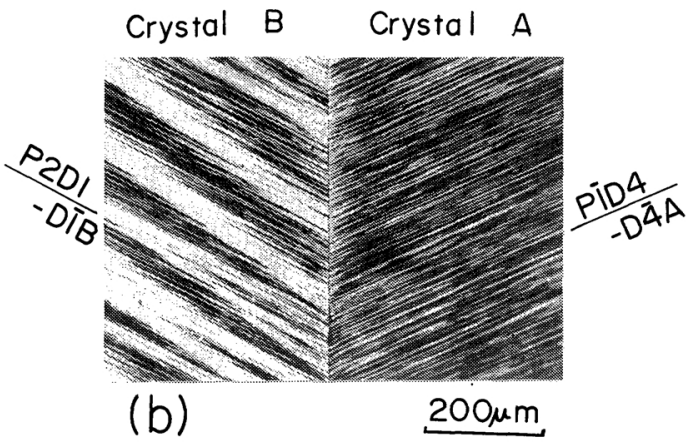

Photo. 7 Slip bands observed at $N=500$ near the crystal boundary in type 1 bicrystal.

(a) The front surface. (b) The back surface.

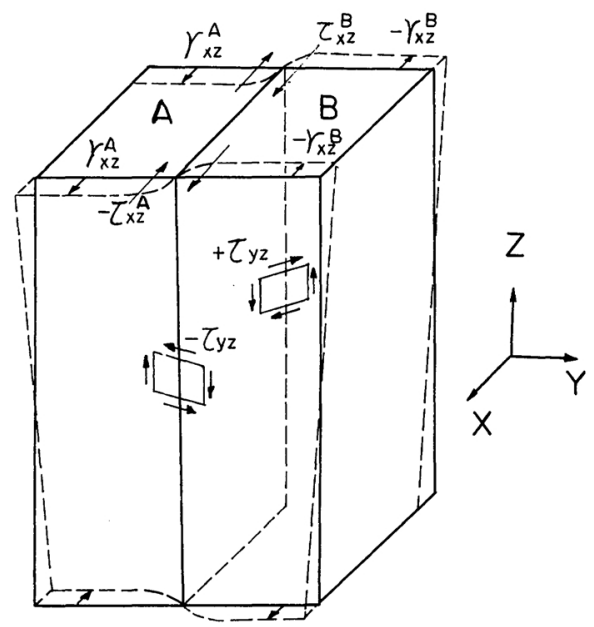

Fig. 6 Schematic illustration showing how stresses $\pm \tau_{x z}$ and $\pm \tau_{y z}$ arise by the incompatibility of elastic strain $\gamma_{x z}$.

the eqs. (1), local shear stresses must be generated near the crystal boundary. Figure $6^{(14)}$ represents how the local shear stresses $\pm \tau_{y z}$ arise from the incompatibility of the elastic strains $\pm \gamma_{x z}$. The absolute values of the local shear stresses $\pm \tau_{y z}$ are maximum at the crystal boundary plane on both surfaces and diminish in proportion to the distance from the crystal boundary or into the interior along the crystal boundary. When the bicrystal is elastically deformed into the shape as shown by the dotted lines in Fig. 6, the local shear stresses $-\tau_{y z}$ and $+\tau_{y z}$ are generated near the crystal boundary on the front and back surfaces, respectively. These $\pm \tau_{y z}$ can be resolved onto all slip systems and promote or constrain these slips. It might, therefore, be possible to infringe on the Schmid law apparently. The orientation factors $N_{i j}$. $\left( \pm \tau_{y z}\right)$ for resolving the shear stresses $\pm \tau_{y z}$ onto each slip system can be calculated by the same method as in the calculation of the more familiar orientation factors $N_{i j}{ }^{(10)}$. The positive values of $N_{i j} \cdot\left( \pm \tau_{y z}\right)$ show the tendency to excite the activation of the corresponding slip systems, while the negative ones have the tendency to prevent it $^{(14)(15)}$.

When a bicrystal (Fig. 1) is pulled toward the direction of $\mathrm{Z}$ axis, the elastic strain components in each component crystal are indicated by the following equations:

$$
\begin{aligned}
& e^{\varepsilon_{x x}}=\mathrm{S}_{13} \sigma_{z z}, e_{e}^{\varepsilon_{y y}}=\mathrm{S}_{23} \sigma_{z z}, e_{e}^{\varepsilon_{z z}}=\mathrm{S}_{33} \sigma_{z z} \\
& { }^{\gamma_{x y}}=\mathrm{S}_{43} \sigma_{z z},{ }_{e} \gamma_{x z}=\mathrm{S}_{53} \sigma_{z z},{ }_{e} \gamma_{y z}=\mathrm{S}_{63} \sigma_{z z},
\end{aligned}
$$

where $\mathrm{S}_{i j}$ are the elastic compliances in each component crystal, and $\sigma_{z z}$ is the normal stress on the XY plane for the direction of the $Z$ axis. The compliances $S_{13}, S_{23}, S_{33}$ and $S_{53}$ are constant in the four types of bicrystals, even though their orientations are changed by rotating them around the $Y$ axis (Fig. 1). It can, therefore, be predicted that the local shear stresses near the crystal boundaries due to the elastic incompatibility might not be generated.

As shown in Photo. 1, the development of the slip bands of P4D3-D $\overline{3}$ B with slip planes parallel to the crystal boundary plane increases with increase of that of the primary slip bands. The $N_{i j}$ values between P4D3-D $\overline{3} \mathrm{~B}$ and the primary slip system P3D3-D $\overline{3} \mathrm{~A}$ in the neighboring crystal $\mathrm{A}$ are small $\left(N_{i j}=0.29\right)$, and the $N_{i j}$ values between P4D3-D $\overline{3} B$ and P$\overline{1} D 4-D \overline{4} A$ are small, too $\left(N_{i j}=0.17\right)^{(8)}$. Therefore, the activation of $\mathrm{P} 4 \mathrm{D} 3-\mathrm{D} \overline{3} \mathrm{~B}$ near the crystal boundaries cannot be explained by considering 
the $N_{i j}$ values.

According to the compatibility requirements of the plastic strains by Livingston and Chalmers ${ }^{(10)}$, let us try to explain the activation of the slip system P4D3-D鸟 with slip planes parallel to the crystal boundary plane. The plastic strain components of main slip systems in type 3 bicrystal are given in Table 2 . The values of the plastic strains ${ }_{p} \varepsilon_{x x}$ and ${ }_{p} \varepsilon_{z z}$ for the unit slip in $\mathrm{P} 3 \mathrm{D} \overline{3} \mathrm{~A}$ are almost equal to those for P1'D4B, respectively. The difference of the values of ${ }_{p} \gamma_{x z}$ between them is relatively large ${ }^{(8)}$. However, such a degree also exists in type 1 and 2 in which no fatigue cracks along the crystal boundaries are initiated. The activation of P4D3-D鸟 can, therefore, not be explicated by considering the compatibility requirements of the plastic strains, either.

Here, it can be recognized that the screw dislocation walls formed on the crystal boundary by the screw dislocations of the primary

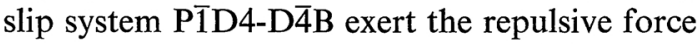
over a long range upon the screw dislocations of the primary slip system P3D3-D 3 A approaching the crystal boundary in type 3 and 4 . As the result of it, the slip band tips of P3D3$\mathrm{D} \overline{3} \mathrm{~A}$ are impeded near the crystal boundaries. In these places, the stress field of the above screw dislocation walls exerts the attrative force over the long range upon the screw dis-

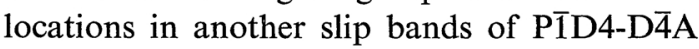
which arrive at the crystal boundary. The

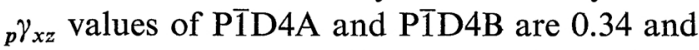
0.02 , respectively. The difference between them is so large. It may, therefore, be considered that the local shear stresses $\pm \tau_{y z}$ is generated near the crystal boundaries so as to diminish the "incompatibility" $\dagger$ of the plastic strains ${ }_{p} \gamma_{x z}$. The absolute values of the stresses $\pm \tau_{y z}$ increase in proportion to the distance to the crystal boundary plane at which they are maximum.

$\uparrow$ In addition to the familiar incompatibility requirements of the plastic strains discussed by Livingston and Chalmers ${ }^{(10)}$, this "incompatibility" of the plastic strains includes the consideration for the interaction between dislocation walls which are formed by the dislocations of the primary slip systems during deformation and approaching dislocations in adjoining crystals ${ }^{(16)}$.
Table 2 Plastic strain components of main slip systems in type 3 bicrystal (under tensile stress).

\begin{tabular}{|c|c|c|c|}
\hline Slip system & ${ }_{p} \varepsilon_{x x}$ & ${ }_{p} \varepsilon_{z z}$ & $p \gamma_{x z}$ \\
\hline P3D $\overline{3} A$ & -0.46 & 0.43 & 0.16 \\
\hline Pर्206A & -0.05 & 0.32 & 0.22 \\
\hline PĪD4A & -0.40 & 0.39 & -0.34 \\
\hline P'̄D1A & -0.07 & 0.09 & 0.47 \\
\hline P̄̄D4B & -0.48 & 0.47 & 0.03 \\
\hline $\mathrm{P} 2 \mathrm{D} \overline{5} \mathrm{~B}$ & -0.12 & 0.38 & 0.14 \\
\hline P2D1̄B & -0.28 & 0.25 & -0.40 \\
\hline $\mathbf{P} 4 \mathrm{D} \overline{3} \mathrm{~B}$ & -0.01 & 0.01 & 0.01 \\
\hline
\end{tabular}

Table 3 Orientation factor $N_{i j}$ for resolved shear stress of $\pm \tau_{y z}$ onto main slip systems in four types of bicrystals (under tensile stress).

\begin{tabular}{|c|c|c|c|c|}
\hline Type & $\begin{array}{c}\text { Slip } \\
\text { system }\end{array}$ & $\begin{array}{l}N_{i j}\left(-\tau_{y z}\right) \\
\text { (the front } \\
\text { surface) }\end{array}$ & $\begin{array}{l}N_{i j}\left(+\tau_{y z}\right) \\
\text { (the back } \\
\text { surface) }\end{array}$ & $\begin{array}{l}\text { Schmid } \\
\text { factor }\end{array}$ \\
\hline \multirow{6}{*}{1} & PĪD4A & -0.06 & 0.06 & 0.47 \\
\hline & P2D $\overline{5} A$ & -0.55 & 0.55 & 0.39 \\
\hline & $\mathrm{P} 4 \mathrm{D} \overline{3} \mathrm{~A}$ & 0.99 & -0.99 & 0.02 \\
\hline & P2DĪB & 0.13 & -0.13 & 0.50 \\
\hline & P2D $6 \bar{B}$ & -0.37 & 0.37 & 0.37 \\
\hline & P4D3B & 0.96 & -0.96 & 0.00 \\
\hline \multirow{6}{*}{2} & P̄̄D4A & -0.21 & 0.21 & 0.47 \\
\hline & P2D $\overline{5} A$ & -0.60 & 0.60 & 0.36 \\
\hline & P4D $\overline{3} A$ & 0.96 & -0.96 & 0.02 \\
\hline & P2D1̄B & 0.19 & -0.19 & 0.44 \\
\hline & $\mathrm{P} 2 \mathrm{D} \overline{\overline{5}} \mathrm{~B}$ & -0.46 & 0.46 & 0.41 \\
\hline & P4D3B & 1.00 & -1.00 & 0.00 \\
\hline \multirow{7}{*}{3} & P'̄D4A & -0.31 & 0.31 & 0.35 \\
\hline & P3D $\overline{3} A$ & -0.25 & 0.25 & 0.43 \\
\hline & P'̄D6A & -0.63 & 0.63 & 0.32 \\
\hline & P4D $\overline{3} A$ & 0.82 & -0.82 & 0.01 \\
\hline & P'̄D4B & -0.24 & 0.24 & 0.47 \\
\hline & P2D $\overline{5} B$ & -0.58 & 0.58 & 0.38 \\
\hline & P4D $\overline{3} B$ & 0.96 & -0.96 & 0.01 \\
\hline \multirow{7}{*}{4} & PT̃4A & -0.30 & 0.30 & 0.19 \\
\hline & P3D $\overline{3} A$ & -0.25 & 0.25 & 0.45 \\
\hline & Pर̄D6A & -0.58 & 0.58 & 0.33 \\
\hline & P4D $\overline{3} A$ & 0.77 & -0.77 & 0.05 \\
\hline & P̄̄D4B & -0.26 & 0.26 & 0.46 \\
\hline & $\mathrm{P} 2 \mathrm{D} \overline{\bar{B}} \mathrm{~B}$ & -0.65 & 0.65 & 0.34 \\
\hline & P4D $\overline{3} B$ & 0.95 & -0.95 & 0.05 \\
\hline
\end{tabular}

Table 3 gives the orientation factors $N_{i j}$. $\left( \pm \tau_{y z}\right)$ for resolving the shear stresses $\pm \tau_{y z}$ onto main slip systems in four types of bicrystals under the tensile stress. The values of 
$N_{i j} \cdot\left( \pm \tau_{y z}\right)$ of P4D3B in types 1 and 2 , and $P 4 D \overline{3} B$ in types 3 and 4 are larger than 0.95 . Therefore, if the local shear stress of $-\tau_{y z}$ is generated near the crystal boundary on the front surface of four types, the slip system P4D $\overline{3} B$ with slip planes parallel to the crystal boundary plane will be activated near the crystal boundary. However, what are the reasons why on the back surface in types 3 and 4 and on either surface in types 1 and 2 the slip system P4D3-D $\overline{3} B$ is not activated?

On the back surface in types 3 and 4 bicrystals, the secondary slip bands of Pर्D6A and $\mathrm{P} 2 \mathrm{D} \overline{\mathrm{B}} \mathrm{B}$ are observed near the crystal boundaries. But they are not activated on the front surface as mentioned previously. The $N_{i j}$ values between these slip systems and each corresponding primary slip system are 0.45 and 0.47 , respectively, whose values are recognized to be considerably large. The $N_{i j} \cdot\left(+\tau_{y z}\right)$ for the resolved shear stress of $+\tau_{y z}$ on these secondary slip systems are 0.63 and 0.58 , and their Schmid factors are 0.32 and 0.38 , respectively. The magnitudes of these values are so large that the slipping of $\mathrm{P} \overline{2} \mathrm{D} 6 \mathrm{~A}$ and P2D $\overline{5} B$ occurs on the back surface. On the front surface, however, the $N_{i j} \cdot\left(-\tau_{y z}\right)$ on these secondary slip systems are -0.63 and -0.58 . This means that; by the occurrence of the local shear stress $-\tau_{y z}$ near the crystal boundaries on the front surface in types 3 and 4, the activation of these systems is prevented, while that of P4D3B is promoted.

On the other hand, in the vicinity of the crystal boundaries on the front and back surfaces in types 1 and 2 the local shear stresses $\pm \tau_{y z}$ might not be so large that the slip system P4D3B would not be activated in spite of the largest $\left|N_{i j} \cdot\left( \pm \tau_{y z}\right)\right|$ values, because the "incompatibility" of the plastic strains is small (cf. Table 1 and Photo. 7). The $N_{i j} \cdot\left( \pm \tau_{y z}\right)$ values under compression stress and tensile stress in a bicrystal are the same. Therefore, the activation of the slip system P4D3-D $\overline{3} B$ with slip planes parallel to the crystal boundary plane near the crystal boundary on the front surface in types 3 and 4 can be promoted by subjecting the fatigue bicrystal specimens to the cyclic bending stress.

According to these discussions we can find a clue to the reasons why the fatigue cracks can be initiated and propagated along the crystal boundaries on a certain surface in certain oriented bicrystals.

\section{Conclusions}

Aluminum bicrystals having a longitudinal crystal boundary parallel to the $\{111\}$ plane and with a twist angle of about $0.38 \mathrm{rad}\left(22^{\circ}\right)$ were grown by a modified Bridgman method. The fatigue tests were done on a bending fatigue testing machine and carried out at a constant strain amplitude of about $0.2 \%$ and cyclic frequencies of $30.4 \mathrm{~Hz}$. The following results were obtained:

(1) It has been found that fatigue cracks are reproducibly initiated and propagated along the crystal boundaries on a certain surface in certain oriented bicrystals. For the initiation and propagation of the fatigue cracks along the crystal boundaries, it is necessary for the slip bands to occur along the crystal boundaries previously.

(2) In order to examine the mechanism of the initiation of the fatigue cracks along the crystal boundaries, it is necessary to take into consideration the compatibility requirements of the plastic strains at the crystal boundaries. Then, we should give attention to the "incompatibility" of the plastic strains at the crystal boundaries including the consideration for the interaction between dislocation walls formed by the dislocations of the primary slip systems and approaching dislocations in adjoining crystals during deformation.

(3) In bicrystals whose slip systems with slip planes parallel to the crystal boundary plane are not activated, other slip systems are activated near the crystal boundary, and any fatigue crack along the crystal boundary are not initiated.

\section{REFERENCES}

(1) For example, P. T. E. Forsyth: Nature, 171 (1953), 72.

(2) G. C. Smith: Proc. Roy. Soc. A, 242 (1957), 189.

(3) D. S. Kemsley: J. Inst. Metals, 85 (1956-57), 420.

(4) C. Laird and G. C. Feltner: Trans. Met. Soc. AIME, 239 (1967), 1074.

(5) W. A. Wood, W. H. Reiman and K. R. Sargant: Trans. Met. Soc. AIME, 230 (1964), 511. 
(6) G. Mima, T. Oka and F. Inoko: J. Japan Inst. Metals, 35 (1971), 646.

(7) F. Inoko, K. Akizono and G. Mima: J. Japan Inst. Metals, 36 (1972), 375, 380.

(8) F. Inoko, H. Yamaji, K. Akizono and G. Mima: J. Japan Inst. Metals, 39 (1975), 467.

(9) F. Inoko, K. Akizono, H. Yamaji and G. Mima: Proc. 2nd Inter. Conf. on Mechnical Behavior of Materials, 16-20 August (1976) Boston, p. 13.

(10) J. C. Livingston and B. Chalmers: Acta Met., 5 (1957), 322.

(11) G. Mima, F. Inoko, K. Atagi and K. Uesaki: J.
Japan Inst. Light Metals, 28 (1978), 130.

(12) R. E. Peterson: Stress Concentration Design Factor, John Wiley, New York, (1974), p. 44.

(13) J. C. M. Li and C. D. Needham: J. Appl. Phys., 31 (1960), 1318.

(14) R. E. Hook and J. P. Hirth: Acta Met., 5 (1967), 535.

(15) T. D. Lee and H. Margolin: Met. Trans., 8A (1977), 145, 157.

(16) S. Kimura, F. Inoko and K. Akizono: Reported on the Meeting of J. Japan Inst. Metals, April (1978), p. 243. 